Ann. Génét. Sél. anim., I975, 7 (3), 321-330.

\title{
ÉTAT PRÉSENT DES CONNAISSANCES SUR LE SYNDROME D'ARTHROGRYPOSE ET DE PALATOSCHISIS (SAP) DANS LE BÉTAIL CHAROLAIS EN FRANGE (')
}

\author{
J.-J. LAUVERGNE \\ Département de Génétique animale, \\ Centre. national de Recherches zootechniques, I. N. R. A., \\ 78350 Jouy en Josas (France)
}

\section{RÉSUMÉ}

L'analyse des résultats de testage sur la descendance en race Charolaise pure pour les taureaux d'insémination artificielle en France depuis 1967 a permis de montrer que plusieurs symptômes anomaliques congénitaux étaient héréditaires. Il s'agit en particulier de la fissure palatine, de l'arthrogrypose des membres et de la cyphose. A ce jour, r 34 taureaux sont connus à partir de leurs I9 421 veaux produits lors des opérations de testage.

On a pu ensuite démontrer que ces symptômes héréditaires appartenaient à un syndrome que l'on a appelé Syndrome d'Arthrogrypose et de Palatoschisis, en abrégé SAP.

Le déterminisme héréditaire de SAP le plus probable semble être de type mendélien, monofactoriel, récessif, à pénétrance incomplète. SAP se caractérise par une expressivité variable : environ $30 \mathrm{p}$. 100 des veaux sont seulement arthrogrypotiques, $70 \mathrm{p}$. roo présentent à la fois la fissure palatine et l'arthrogrypose qui sont les deux localisations qui suffisent à déceler le syndrome.

La fréquence du gène et sa pénétrance chez les homozygotes ont été estimés par une analyse des fratries de demi-germains selon une méthode donnée par LAUVERGNe et LEFORT (1973). Pour l'application de ces formules on a dû admettre que le gène avait atteint une fréquence d'équilibre dans la population d'où étaient tirés au hasard quant à leur génotype en SAP les mâles testés et les femelles auxquelles ils ont été accouplées ce qui semble assez raisonnable d'après l'examen de certains indices.

Dans ces conditions, la fréquence $q$ du gène dans la population Charolaise française inscrite au livre généalogique serait comprise entre 0,2 et 0,202 . La pénétrance $w$ chez les homozygotes serait comprise entre $0, I_{1} 5$ et $0,1_{3}$ I entre sexes.

(1) Cette note reprend avec quelques modifications de détail le texte d'une conférence prononcée le 4 juillet 1975 devant le Comité Technique de la Fédération internationale des Associations d'Éleveurs de la race bovine Charolaise (F.I.A.E.R.B.C.), réuni à Calgary, Alberta, Canada. Un résumé, en ang lais, de cette intervention a récemment paru dans Canadian Charolais Banner (LAUvergne, I975). 
Aux États-Unis et au Canada, un syndrome analogue a été étudié en race Charolaise. Il semble qu'il s' agisse du même gène qui n'aurait pas, toutefois, la même pénétrance au Canada qu'en France; il est vrai qu'au Canada, cette pénétrance n'a, jusqu'à présent été estimée que sur des veaux croisés alors qu'en France nos analyses sont faites en race pure.

D'un point de vue génétique l'existence d'un équilibre pour la fréquence du gène en France impliquerait un avantage sélectif des hétérozygotes. Un tel avantage sélectif semble avoir été observé pour les qualités reproductrices des femelles hétérozygotes au Canada. Nous ne l'avons pas vérifié en France, pour les débuts de carrière des femelles du moins avec l'information que nous possédons.

L'éradication en France n'est peut-être pas économiquement justifiée, même si elle l'est commercialement. Il n'y a d'ailleurs pas encore de politique d'éradication vraiment générale. Du côté du cheptel inscrit tout d'abord rien n'a été encore entrepris systématiquement. De toute façon dans les conditions actuelles de l'élevage (petits troupeaux en monte naturelle) l'éradication y semble difficile. La chose est plus facile pour les taureaux soumis au testage sur leur descendance en vue d'être utilisés en insémination artificielle. Ainsi, depuis huit ans $a-t$-on pu détecter et éliminer un certain nombre de transmetteurs du gène.

A terme, toutefois, l'emploi généralisé de semence indemne n'est pas entièrement satisfaisant car la fréquence du gène chez les femelles support de testage va diminuer, rendant la détection de plus en plus difficile.

On a quelques raisons de penser que, dans les rameaux étrangers du Charolais, la fréquence du gène responsable de SAP est du même ordre qu'en France.

\section{INTRODUCTION}

L'étude des défauts congénitaux dans la race Charolaise en France a commencé en r966. A part au tout début (LAUVERGNe et BLIN, 1967), elle est essentiellement basée sur l'analyse de données provenant du contrôle systématique des anomalies des veaux de testage sur la descendance.

Cette analyse a été reprise chaque année, à mesure de l'arrivée des données de la dernière campagne de testage. Ainsi, au fil des ans, l'interprétation du phénomène a-t-elle pu évoluer.

Considérant qu'il s'agissait d'un syndrome à déterminisme héréditaire mendélien monofactoriel nous avons, tout d'abord, hésité entre un comportement dominant et un comportement récessif du gène (LAUvergne et Blin, 1967). Analysant les résultats de la première campagne de testage (1966-1967) nous avons conclu provisoirement que le comportement récessif était le seul acceptable (LAUvergne et Blin, I968). Par la suite trois notes ont été présentées, en I970 et 197r, à une commission nationale ad hoc créée par le Ministère de l'A griculture français pour faire le point sur la question (LAUVERGNe, I970 $a, b$, 1971). Dans la dernière de ces notes on tenait compte d'une pénétrance assez faible chez les homozygotes récessifs. La conférence donnée en 1972 à Kinsella (au Ranch expérimental de l'Université d'Edmonton) devant des scientifiques et des responsables du Charolais canadiens reprenait cette conception (LAUVERGNE, 1972).

En fait, en présence de symptômes nombreux et diversement associés parmi les individus et parmi les descendances de testage, la monofactorialité récessive restait une sorte de postulat, tant qu'une analyse statistique rigoureuse n'avait été menée. Notre effort dans les années 1973 et 1974 a donc logiquement porté sur la mise au point de méthodes, non seulement pour calculer les paramètres génétiques dans une situation non encore explorée par les anciens auteurs (fratries de demi-germains ( ${ }^{1}$ ) avec pénétrance incomplète : LAUVERGNe et LEFoRT, 1973) mais, aussi, pour montrer sans équivoque que l'on pouvait parler d'un syndrome mendélien récessif (LAUVERGNE, 1974).

Actuellement la vérification des données est encore en cours, de même que les calculs définitifs, et un certain nombre de résultats chiffrés doivent encore être considérés comme provisoires. On peut cependant, d'ores et déjà, présenter une série de faits assez bien établis.

(1) En langage de génétique des populations une fratrie est un groupe d'apparentés ayant en commun au moins un parent. Les demi-germains ont la particularité d'avoir en commun le père ou la mère, chez les bovins c'est en général le père du fait de l'insémination artificielle. 


\section{I. - L'ORIGINE DE L'INFORMATION}

Les résultats que nous avons analysés ici proviennent du testage sur la descendance en race Charolaise pure effectué en France par l'Union des Coopératives d'Élevage adhérentes de l'Association de testage de la race Charolaise (U.C.A.A.T.R.C.) qui couvre essentiellement l'Allier, les deux départements berrichons, la Vendée, le Loiret et l'Yonne. A cette union s'est adjointe depuis quelques années l'Union régionale des C.E.I.A. "Centre-Est ": Loire et Saône-et-Loire. La coordination des opérations de testage est assurée par la section d'Amélioration génétique de l'Institut technique de l'Élevage bovin (I.T.E.B.). Les observations systématiques concernant les anomalies ont été, en particulier, faites par les techniciens de cet Institut.

Pour l'interprétation qui va suivre nous disposions des données provenant de huit séries annuelles de testage (de 1967 à 1974 ) et relatives à $\mathbf{1} 34$ taureaux mis à l'épreuve sur leur descendance. Lors de cette épreuve chaque taureau a procréé environ 150 veaux (au total 1942 I), la plupart dans des élevages différents. Si les taureaux proviennent d'élevages inscrits au Herd-Book Charolais les vaches supports utilisées, par contre, ne sont en général pas enregistrées au Livre généalogique de la race. Elles appartiennent à des petits éleveurs qui utilisent largement l'insémination artificielle. Les éleveurs de troupeaux pedigrees ayant fourni les taureaux ont, quant à eux, la plupart du temps leur propre taureau et, de ce fait, ont peu recours à l'insémination artificielle.

\section{2. - LA MISE EN ÉVIDENCE DE I'HÉRÉDITÉ DE CERTAINS SYMPTÔMES}

Plus d'une centaine ( $\mathrm{r}$ ro) de ces veaux de testage se sont révélés anormaux, avec des symptômes très divers dans leur localisation, leur intensité et leur association. Notre première démarche a été de mettre en évidence ceux des symptômes qui étaient héréditaires.

Le test statistique utilisé, décrit par LAUVERGNE (1974) consistait à voir comment chaque localisation anomalique se distribuait parmi les lots de testage. S'il y a distribution au hasard (c'est-à-dire selon une loi de Poisson, car les fréquences sont faibles) on ne peut parler de déterminisme héréditaire. Dans le cas contraire on peut conclure que les pères diffèrent entre eux quant à leur capacité de transmettre le défaut à leur descendance, c'est-à-dire que la tare est à déterminisme héréditaire.

Le test a été appliqué aux trois localisations anomaliques les plus fréquentes et les plus sérieusement notées par les contrôleurs : la fissure palatine, les défauts articulaires des membres et les déformations de la colonne vertébrale. Les analyses permettent de conclure que, dans les trois cas, la distribution du nombre des anormaux dans la descendance des taureaux de testage n'obéit pas à une loi de Poisson (les $\chi^{2}$ sont respectivement de 3209,4 ; I 089,6 et 15, I pour des valeurs de 8,$5 ; 16,8$ et 9,2 au seuil $P=0,01$ ).

L'intérêt de ce résultat n'est pas purement académique. Des défauts comme la fissure palatine ou l'arthrogrypose des membres peuvent en effet avoir chez les Ruminants une étiologie non héréditaire. La tératogenèse par ingestion de plantes vénéneuses par des vaches gestantes n'a pas encore été décrite en France mais elle l'a été aux États-Unis (Brnns, 1972). Quant à certains drogages sur des femelles pleines on peut déjà, dans notre pays, les rendre responsable de cas de tératogenèse exogène, au moins chez la brebis (LApras et al., 1973). Il y a, en outre, l'action possible de virus comme celui qui cause la maladie de la langue bleue (Shimshony, 1970). 


\section{3. - LE REGROUPEMENT DES SYMPTÔMES EN UN SYNDROME D'ÉTIOLOGIE MENDELIENNE : SAP}

Pour tenter, ensuite, de réunir ces symptômes en un syndrome relevant d'une étiologie unique on a utilisé un raisonnement probabiliste par l'absurde. Il s'agit de montrer que toutes les hypothèses hormis la plus simple (un seul facteur mendélien à effets pléïotropiques) ont une probabilité extrêmement faible de s'être réalisées.

La chose est assez facile pour les symptômes les plus fréquents, vu la manière dont ils sont associés dans la descendance des différents taureaux. Considérons par exemple, les deux localisations : au niveau du palais et des membres, et supposons que chacune est sous la dépendance d'un gène distinct. Comme ces symptômes apparaissent dans la descendance de la plupart des taureaux transmetteurs décelés il faut alors admettre que tous ceux-ci portent les denx gènes. En outre les symptômes étant fréquemment associés chez le même individu les deux gènes doivent être sur le même chromosome et en position de couplage. Cela implique que les deux loci aient muté en même temps et assez récemment pour que le jeu du crossing-over n'ait pas eu le temps de les séparer. Or, la probabilité que deux loci très proches mutent en même temps chez le même individu est très faible; par ailleurs les relations de parenté relativement lâches entre les transmetteurs ne permet pas de penser que ces mutations sont récentes. On peut donc rejeter l'hypothèse bifactorielle et, à plus forte raison, tout autre hypothèse plus compliquée et considérer comme la plus vraisemblable l'hypothèse d'un seul mutant responsable, à la fois de la fissure palatine et des anomalies des membres.

Un raisonnement analogue permet de rattacher au même syndrome les atteintes vertébrales.

Ainsi est-il possible de dégager l'existence d'un syndrome à étiologie héréditaire mendélienne monofactorielle que nous avons nommé Syndrome d'Arthrogrypose et de Palatoschisis, en abrégé SAP.

Ce syndrome se manifeste chez le veau nouveau-né en premier lieu par la fissure palatine et/ou l'arthrogrypose des membres.

En fait, au moins dans nos données, la fissure palatine n'allait pratiquement jamais sans les atteintes des membres. Ces dernières se présentaient en l'absence de la fissure, dans environ $30 \mathrm{p}$. Ioo des cas. Les autres atteintes, y compris les anomalies vertébrales, ne se rencontrant jamais hors de la présence de la fissure ou de l'arthrogrypose, à la rigueur elles ne sont pas indispensable pour l'identification du syndrome. Ce sont surtout : une myodystrophie (musculature sous développée), une campyloprosopie (assymétrie de la face), une forme de schisostomie débutante (nombril ouvert) et une forme d'hydrocéphalie.

Quatre-vingt-neuf pour cent des veaux anormaux observés au cours du testage pouvaient être classés comme SAP.

Nos observations ont montré, en outre, que les veaux SAP naissaient à terme, vivants. Ils étaient plus légers que les veaux normaux et moins sujets à des accidents de parturition. Les éleveurs pratiquent sur eux la plupart du temps l'euthanasie mais quelques essais d'élevage ont pu néanmoins être faits. Ils ont montré que bien peu d'anormaux étaient capables de vivre plus de quatre mois.

\section{4. - RÉCESSIVITÉ, PÉNÉTRANCE ETT FRÉQUENCE DU GÈNE RESPONSABLE DE SAP}

Grâce à certaines considérations déjà évoquées (LAUvergne et BLIN, 1968) on peut penser que le gène responsable de SAP a un comportement uniquement récessif ou que sa dominance 
est à pénétrance très basse : aucun cas de SAP signalé au croisement industriel avec des taureaux Charolais réputés transmetteurs.

On peut ainsi utiliser les formules écrites par LAUvergne et Lefort (r973) pour l'analyse des fratries de demi-germains que constituent les lots de testage des différents taureaux. Il faut toutefois, au préalable, que soient vérifiées leurs conditions d'application : état d'équilibre de la fréquence génique dans une population d'où sont issus les reproducteurs sans que ceux-ci soient le moins du monde choisis en fonction de leur génotype SAP.

L'examen de la fréquence des transmetteurs détectés ( $\left.{ }^{1}\right)$ au Centre d'insémination de l'Allier entre 1957 et 1965 et, depuis cette date, parmi les taureaux mis au testage, permet de soupçonner fortement qu'un équilibre de la fréquence génique de SAP s'est établi depuis longtemps dans la race (au moins une trentaine d'années). Quant au choix des reproducteurs mâles testés on a pu voir qu'aucun critère évident se rapportant à leur génotype en SAP n'avait été retenu lors de leur sélection. Par ailleurs l'échantillonnage porte sur pratiquement toute l'aire d'expansion de la race, ce qui permet de penser que l'on échappe à tout effet de dérive génétique.

Quant à la population des femelles inséminées par ces mâles de testage, le développement ancien de l'insémination (25 ans environ) dans les élevages d'où elles sont issues conduit à accepter l'hypothèse selon laquelle pour la présence du gène SAP elles constituent une population voisine de celle des mâles inscrits mis à l'épreuve sur leur descendance. Sur les données déjà disponibles nous avons alors appliqué les formules simplifiées en tenant compte de deux situations : celle où tous les cas encore en cours de vérification pour la paternité et la présence du syndrome sont omis (hypothèse basse) et celle où tous c es cas sont conservés (hypothèse haute).

\section{TABIEAU I}

Estimation des paramètres génétiques relatifs à SAP dans le Charolais français

\begin{tabular}{|c|c|c|c|c|}
\hline & & \multirow{2}{*}{$\begin{array}{l}\text { Estimations } \\
\text { de LAUVERGNE } \\
(1972)\end{array}$} & \multicolumn{2}{|c|}{ Présentes estimations (1975) } \\
\hline & & & $\begin{array}{l}\text { hypothese } \\
\text { basse }\end{array}$ & $\begin{array}{l}\text { hypothese } \\
\text { haute }\end{array}$ \\
\hline \multirow[t]{2}{*}{ Effectifs } & $\begin{array}{l}\text { - taureaux testés. } \ldots \ldots \ldots \ldots \ldots \ldots \ldots \ldots \\
\text { - veaux observés } \ldots \ldots \ldots \ldots \ldots \ldots \ldots\end{array}$ & $\begin{array}{r}99 \\
15775\end{array}$ & \multicolumn{2}{|c|}{$\begin{array}{r}134 \\
19421\end{array}$} \\
\hline & - taureaux transmetteurs détectés .... & 30 & 33 & 43 \\
\hline Fréquence & $\begin{array}{l}\text { - transmetteurs détectés } \ldots \ldots \ldots \ldots \ldots \\
\text { - transmetteurs prévus par le calcul } \ldots \\
\text { - gène SAP }(q) \ldots \ldots \ldots \ldots \ldots \ldots \ldots\end{array}$ & $\begin{array}{l}0,303 \\
0,356 \\
0,20\end{array}$ & $\begin{array}{l}0,246 \\
0,360 \\
0,200\end{array}$ & $\begin{array}{l}0,320 \\
0,363 \\
0,202\end{array}$ \\
\hline \multicolumn{2}{|r|}{ Taux de détection des porteurs } & $85 \%$ & $68 \%$ & $88 \%$ \\
\hline Pénétrance & $\begin{array}{l}w_{m} \text { chez les mâles homozygotes........ } \\
w_{f} \text { chez les femelles homozygotes...... } \\
w \text { entre sexes } \ldots \ldots \ldots \ldots \ldots \ldots \ldots \ldots\end{array}$ & $\begin{array}{l}0,14 \\
0,10\end{array}$ & 0,115 & 0,131 \\
\hline
\end{tabular}

On obtient alors pour $q$ (fréquence du gène supposée à l'équilibre), $w_{m}$ (pénétrance chez les mâles) et $w_{f}$ (pénétrance chez les femelles), les estimées qui sont données dans le tableau I. Dans ce même tableau on a également présenté les estimations présentées en 1972 (LAUVERGNE, 1972).

L'écart entre les estimations de 1972 et les présentes estimations est négligeable. Par

(1) Un transmetteur détecté est un reproducteur qui a eu au moins un SAP dans sa descendance. 
contre l'écart avec les estimations données plus récemment (LAUvergne, r975) est notable mais sans importance car ces récentes estimations, faites sans passage sur ordinateur, à partir d'une équation simplifiée ne doivent plus être prise en considération.

Bien entendu la valeur estimée de la pénétrance qui parait différer selon le sexe dépend étroitement de la finesse de la détection des tarés. Notre méthode de simple examen visuel peu après la naissance par des techniciens non spécialisés en pathologie, est certainement incomplète. A cet égard les articles de Giroud (1972) et Giroud et Gueguen (1973) dans la zone Charolaise témoignent de l'existence d'anomalies plus frustres des membres qui pourraient fort bien correspondre à une action moins spectaculaire mais tout aussi réelle du gène étudié.

Pour l'instant il faut retenir que la fréquence du gène est relativement élevée dans le Charolais français mais que la pénétrance (limitée aux cas extrêmes) est très basse. Aussi n'observe-t-on qu'une fréquence d'environ 0,4 p. Ioo de tarés, pratiquement inviables.

\section{5. - IA SITUATION EN RACE CHAROLAISE, DANS LES AUTRES PAYS}

SAP tel que nous l'observons en France est très semblable à des syndromes dont l'existence est signalée un peu partout dans le monde du Charolais mais qui n'ont encore été décrits en détail qu'aux États-Unis (Greeley et Jolly, 1970, Greeley et al., 1970) et au Canada (Leipold et al 1969, 1970).

Les auteurs canadiens du Saskatchewan considéraient déjà qu'il s'agissait d'un syndrome mendélien récessif à pénétrance complète, (Howell et Leipold (I970), NAwrot (I973). BERG et GOODEWARENE (1974) à partir de données expérimentales receuillies au ranch de Kuisella aboutissent aux mêmes conclusions.

En raisonnant par l'absurde comme précédemment, il semble possible d'avancer qu'il s'agit, des deux côtés de l'Atlantique des effets du même gène apparu en France dans la population Charolaise avant les premières exportations qui, pour l'Amérique du Nord, remontent à $193^{\circ}$ (ANonyme, 1973). Il n'en reste pas moins que le comportement du gène, si l'on en juge par la pénétrance chez l'homozygote, diffère considérablement entre la France et le Canada.

Au Canada comme la plupart des cas de SAP se rencontrent chez des veaux $3 / 4$ ou $7 / 8$ de sang Charolais, Berg et Goonewardene (1974) pensent que ces veaux ne possèdent pas toutes la panoplie des modificateurs qui, en race pure, sont capables de réduire si fortement la pénétrance. Mais cette explication sous-entend l'existence d'un effet de seuil. Toutefois on peut également penser que les méthodes utilisées par Berg et Goodewarene (1974) et par NaWrot (1973) sont partiellement biaisées dans le choix même des fratries : l'analyse ne porte que sur les fratries où la combinaison des modificateurs favorise l'expression de la tare, donc la pénétrance pourrait être, par cette méthode, surestimée.

\section{6. - LES RAISONS DU MAINTIEN A L'ÉQZUILIBRE DE LAA FRÉQUENCE GÉNIQUE}

Dans le cas de biallélisme, pour observer un équilibre stable et non nul des fréquences géniques il faut et suffit que la valeur sélective de l'hétérozygote soit supérieure à celle des deux homozygotes. Dans ce cas il se produit ce qu'on appelle un polymorphisme balancé d'origine ségrégationnelle. C'est cette explication qui prévaut généralement en génétique des populations humaines où ces phénomènes d'équilibre ont été spécialement étudiés (cf. LaLouEL, r970 pour revue). Ayant 
acquis une raisonnable certitude que la fréquence du gène SAP avait atteint en France une fréquence d'équilibre (la proportion des mâles de testage transmetteurs détectés se maintenant d'année en année), nous avions suggéré qu'un avantage des hétérozygotes SAP devait exister ; nous pensions surtout à une meilleure fertilité des femelles (LAUvergne, 1972). En France, à la Station d'Agonges, dans l'Allier où l'on teste les qualités d'élevage des filles des taureaux de race Charolaise, nous avons pu comparer certaines performances de reproduction (précocité, sexuelle, fécondité à la première insémination, pourcentage de veaux sevrés, difficulté de vêlage, aptitude maternelle) entre groupes de filles issues de taureaux non porteurs et de taureaux transmetteurs détectés. Aucune différence évidente n'a pu être décelée. Par contre BERG et GoOdEWARDENe (1974), à partir d'observations faites sur le troupeau expérimental de l'Université d'Edmonton (Alberta) au ranch de Kinsella déjà cité ont trouvé, chez les femelles transmettrices, des qualités reproductrices nettement supérieures à celles des femelles normales : taux de gestation supérieur et âge moyen à la réforme plus élevé. La discordance entre les observations en France et au Canada n'est toutefois pas définitive: les données françaises ont trait au début dela vie sexuelle alors qu'au Canada on a suivi les femelles seulement après qu'elles aient commencé leur carrière de reproductrices.

\section{7. - L'ÉRADICATION EN FRANCE}

En admettant que les femelles hétérozygotes ont aussi en France un avantage sur les femelles normales, l'éradication du gène, du strict point de vue de la rentabilité de la production de veaux sevrés, ne se justifierait pas dans notre pays et cela d'autant moins que les pertes actuelles sont seulement de 0,4 p. roo des veaux. Toutefois, si l'on raisonne en fonction de la diffusion commerciale du Charolais, cette conclusion ne tient pas forcément.

La politique d'éradication suivie jusqu'à présent en France est le reflet de cette ambiguité et des difficultés pratiques de mise en place. Elle varie selon les situations. C'est ainsi qu'il n'y a pas de programme spécifique d'éradication dans le troupeau inscrit alors que, dans le cadre des opérations de testage en race pure des taureaux destinés à l'insémination artificielle on élimine la plupart des mâles transmetteurs détectés.

De fait l'éradication dans le cheptel inscrit, réparti en de multiples troupeaux qui pratiquent surtout la monte naturelle, ne peut avoir qu'une efficacité limitée, même si l'on fait abstraction des obstacles psychologiques. En effet la détection des mâles transmetteurs y est mauvaise car le nombre de leurs descendants est faible. On peut voir aisément que, dans l'hypothèse basse, un transmetteur hétérozygote a une chance sur deux de ne pas être détecté s'il a seulement 76 descendants. Or ce chiffre n'est bien souvent pas atteint quand le taureau a fini sa carrière dans un élevage privé.

Quant à l'éradication dans le cadre des opérations de testage des taureaux destinés à l'IA, elle se fait avec une assez bonne précision. Les taureaux non détectés lors du testage peuvent l'être plus tard à l'aide d'un système de repérage des tarés qui se perfectionne d'année en année. Toutefois, avec la mise en service de plus en plus de taureaux indemnes, la fréquence du gène dans le troupeau des femelles non inscrites va baisser et la détection risque de devenir de moins en moins précise, sans que cela profite à l'ensemble de la population inscrite, qui utilise encore très peu l'insémination artificielle.

\section{8. - L'ÉRADICATION A L'ÉTRANGER}

Nous avons vu plus haut que, selon toute probabilité, la gène responsable de SAP était apparu il y a assez longtemps en France. Il avait déjà bien diffusé dans la race avant que ne commencent les exportations massives. Cela veut dire qu'un peu partout dans le monde sa fréquence 
pourrait être assez voisine de ce qu'elle est en France, avec des inconvénients qui varient selon les plans de croisement.

Les Canadiens semblent actuellement avoir un programme d'éradication assez élaboré. Il consiste d'abord en une identification et un recueil des veaux SAP pour dissection au Western College of Veterinary Medecine de Saskatoon. En outre un troupeau de femelles transmettrices a été constitué et sert déjà à détecter le génotype des mâles mis au testage. Enfin une liste des mâles transmetteurs détectés est tenue à jour et communiqué, sous certaines conditions (QUANrz, 1975).

\section{9. - CONCLUSIONS}

Une telle situation d'équilibre ségrégationnel d'un gène d'anomalie n'est pas absolument absente chez les Bovins domestiques : on connaît par exemple le blanc du Shorthorn et l'achondroplasie du Dexter. L'originalité ici, réside peut-être dans la très faible valeur de la pénétrance.

De nombreux aspects du comportement du gène restent cependant encore dans l'ombre, en particulier chez les homozygotes porteurs sains. La question de l'éradiction aussi bien dans son principe même que dans ses modalités reste également loin d'être entièrement résolue.

Rę̧u pour publication en août 1975.

\section{REMERCIEMENTS}

P. FABRÈGue, responsable du testage charolais à l'I.T.E.B., a eu la difficile tâche de réunir les données qui ont servi à cette étude.

B. VISSAC et P. MÉnissier ont relu le manuscrit et suggéré d'utiles corrections.

\section{SUMMARY}

\section{PRESENT STATE OF KNOWLEDGE \\ ON THE SYNDROME, OF ARTHROGRYPOSIS AND PALATOSCHISIS (SAP) \\ IN FRENCH CHAROLAIS CATTLE}

A statistical study of the results of the progeny testing program performed in French Chaolais cattle in the frame of A.J. has first shown that several congenital symptoms were hereditary, mainly cleft palate, arthrogryposis and cyphosis. The progeny of 134 bulls was examined : 19421 calves.

These hereditary symptoms were, further on, demonstrated to make up one unique Syndrome : the Syndrome of Arthrogryposis and Palatoschisis (SAP).

The most probable determinism of this hereditary syndrom is monofactorial, mendelian, recessive with incomplete penetrance.

The expressivity of SAP is variable. Considering only the two main localisations (palate and legs) which are sufficient to detect its presence one sees that $30 \mathrm{p}$. 100 of calves are only arthrogrypotic while $70 \mathrm{p}$. Ioo present both impairements.

Gene frequency and penetrances have been estimated by half-sib analysis according a newly set-up method (LEFORT and LAUVERGNe, 1973).

Admitting with some good reasons that the gene frequency is in an equilibrium state in the population where males and females are random mated according to their SAP genotype the gene frequency in the pedigree French Charolais could be estimated between .20 and .202. The between sex estimation of penetrance in homozygous $(w)$ is between .I3I and .II5. 
In U.S.A. and Canada the presence of SAP seems also have been demonstrated. Probably it is the same genic etiology. Nevertheless its behaviour appears different in France and in Canada. In this last country the penetrance seems close to Ioo p. I00 when it is much lower in France (see above). But the estimations in Canada are made pratically only on $3 / 4$ blood Charolais calves as in France we worked in pure breed.

The fact that the gene frequency in France is balanced implies heterozygous selective advantage, at least in one sex. This selective advantage seems to have been demonstrated in Canada for reproductive qualities of heterozygous females. In France we have, up to now, no detected a difference but the analysis were only on the beginning of the reproductive career of females.

Eradication in France would probably not be economically justified in any case. Actually, moreover, there is not a general eradication policy.

As concerns the pedigree herds, nothing as yet has been done systematically. Anyway, in present breeding conditions (small units using natural mating) eradication would seem difficult. It is easier among progeny-tested bulls where abnormal progeny are controlled. Thus, for 8 years, most of the carriers of the gene have been eliminated among the I 34 bulls tested in the above mentionned program. However utilization of such a semen free of SAP gene may not be a satisfactory solution for the future because gene frequency will be lowered in inseminated females, thus making detection increasingly difficult. In the meanwhile the frequency in the herdbook population, where insemination is little practised, may continue a long time still.

There are good reasons to believe that, in foreign Charolais branches, the situation of the SAP gene frequency is the same as in France because the appearance and diffusion of this factor seem to go back to the period before large exportation.

\section{RÉFÉRENCES BIBLIOGRAPHIQUES}

Anoṇme, r973. La race Charolaise, I. Résultats étrangers. I.N.R.A., Département de génétique animale, Jouy en Josas, 9-rg.

Berg R. T., Goonewardene L. A., 1974. The genetics of Arthrogryposis in Charolais cattle. Ier Congr. mond. Génét. appl. Élev., 1, 635-642.

Binns W., 1972. Syndrome de la déformation générale du squelette chez le veau. Le Charolais (25), 62-64.

GrRoud A., I972. Syndromes arthro-myodysplasiques et leur cause chez l'homme et l'animal. Bull. Acad. Méd., 156, 814-815.

Giroud A., Gueguen L., 1973. Sur les arthromyodysplasies chez le veau. Bull. Acad. Vét., 96, 443-446.

Greeley R. G., Boyd C. L., Jolly D. G., I968. Bovine anomalies and spastic paresis. Southwest. Vet., 12, 277-280.

Greeley R. G., Jolly D. G., r968. A clue to the etiology of spastic paresis. Southwest Vet., 12, I89-I9I.

Howell W. E., Leipold H. W., r97o. An inherited defect in newborn Charolais calves. Fédération internationale des éleveurs de la race bovine Charolaise, Londres, 2 juillet 1970,3 p., ronéoté.

LALOUEL J.M., 1970. Concentrations locales d'affections héréditaires rares. L'Expansion scientifique française, Paris.

Lapras M., Deschanel J. P., Delatour P., Gastellu J., Lombard M., ig73. Accidents tératologiques chez le mouton après administration de Parbendazole. Bull. Soc. Sci. Vét. Méd. comp., Lyon, 75, 53-6r.

Lauvergne J. J., I97o $a$. Point des connaissances actuelles. In J.M.D. Réunion du 9 juin 1970 du Groupe d'étude du caractère "Palais fendu" en Charolais. Section Amélioration génétique. I.T.E.B., Paris, ronéoté, $6 \mathrm{p}$.

LAUVERGNE J. J., I97o $b$. Quelques considérations sur la fissure palatine en race Charolaise. In J.M.D. Réunion du 9 juin 1970 du Groupe d'étude du caractère "Palais fendu " en Charolais. Section Amélioration génétique. I.T.E.B., Paris, suppl. ronéoté, 2 p.

LAUVERGNe J. J., I97I. Déterminisme héréditaire du syndrome et conséquences pour la sélection du Charolais. Réunion du groupe d'étude "Palais fendu "en Charolais du 13 octobre 1971. Section Amélioration génétique. I.T.E.B., Paris, 2 p. ronéoté.

LAUvergne J.J., I972. Hereditary determinisms of arthrogryposis with palatoschisis syndrome in Charolais cattle. Conférence donnée le 20 juin 1972 au Ranch expérimental de l'Université d'Edmonton Kinsella, Alberta, non publiée (Certains résultats et interprétations sont cités par Berg et GoOdeWARdENe (1974)).

LAUvergne J. J., I974. Étude des gènes à effets visibles à partir des données de testage des taureaux sur leur descendance. I ${ }^{\mathrm{er}}$ Congr. mond. Génét. appl. Elev., 1, 47-55. 
Lauvergne J. J., 1975. Present state of knowledge on the syndrome of anthrogryposis and palatoschisis (SAP) in french Charolais cattle. Technical comittee World Federation of Charolais, July 5 th, I975, Calgary, p. I6, roneotyped.

Lauvergne J. J., Blin P. C., I967. Fissure palatine héréditaire associée à l'ankylose des membres dans la race Charolaise. Ann. Zootech., 16, 291-300.

Lauvergne J. J., Blin P. C., r968. Hereditary determinism of the cleft palate associated with ankylosis of limbs in Charolais cattle. XII int. Conf. Genet., 1, 277.

Lauvergne J. J., Lefort G., 1973. Nouvelle méthode pour analyser le comportement de la fréquence des gènes récessifs à effets visibles dans les populations bovines. C. R. Acad. Sc., Paris (série D), 27\%, 2793-2796.

Leipold H. W., Cates W. F., Radostits O. M., Howell W. E., I969. Spinal dysraphism, arthrogryposis and cleft palate in newborn Charolais calves. Can. vet. J., 10, 268-273.

Leipold H. W., Cates W. F., Radostits O. M., Howell W. E., r97o. Arthrogryposis and associated defects in newborn calves. Am. J. vet. Res., 31, 1367, 74.

Nawrot P., I973. Arthrogryposis, a congenital defect in newborn calves. M. S. Thesis, Dpt. of Anim. Sci. Univ. Saskatchewan, Saskatoon, pp. 138, ronéoté.

Quantz L. I975. C.C.A. newsletter. Can. Charolais Banner, 9 (7), 78.

Shrmshony A., r97o. Blue tongue in cattle, a review. Refuah Vet., 27, 36-42. 